\title{
InP-based planar photonic crystals infiltrated with solid polymers and liquid crystals
}

\author{
Rob van der Heijden ${ }^{\text {aa, }}$, Charlotte Kjellander ${ }^{\mathrm{b}}$, Carl-Fredrik Carlström ${ }^{\mathrm{a}, \mathrm{b}}$, Juri Snijders ${ }^{\mathrm{a}, \mathrm{b}}$, \\ Harm Kicken ${ }^{\mathrm{a}, \mathrm{b}}$, Rob W van der Heijden ${ }^{\mathrm{a}, \mathrm{b}}$, Cees Bastiaansen ${ }^{\mathrm{c}}$, Dick Broer ${ }^{\mathrm{c}}$, Fouad Karouta ${ }^{\mathrm{a}}$, \\ Richard Nötzel ${ }^{\mathrm{a}, \mathrm{b}}$, Emile van der Drift ${ }^{\mathrm{d}}$ and Huub Salemink ${ }^{\mathrm{a}, \mathrm{b}, \mathrm{d}}$ \\ ${ }^{a}$ COBRA Inter-University Research Institute and Center for NanoMaterials, \\ Eindhoven University of Technology, PO Box 513, NL 5600 MB Eindhoven, The Netherlands. \\ ${ }^{b}$ Department of Applied Physics, Eindhoven University of Technology, P. O. Box 513, \\ NL 5600 MB Eindhoven, The Netherlands \\ ${ }^{c}$ Department of Chemistry and Chemical Engineering, Eindhoven University of Technology, \\ P. O. Box 513, NL-5600 MB Eindhoven, The Netherlands \\ ${ }^{\mathrm{d}}$ Kavli Institute of Nanoscience, Delft University of Technology, P.O. Box 5053, \\ NL-2600 GB Delft, The Netherlands.
}

\begin{abstract}
The filling is reported of the air holes of an InP-based two-dimensional photonic crystal with solid polymer and with liquid crystal $5 \mathrm{CB}$. The polymer filling is obtained by thermal polymerization of an infiltrated liquid monomer, trimethylolpropane triacrylate. The filling procedure for both the monomer and liquid crystal relies on the capillary action of the liquid inside the $\sim 200 \mathrm{~nm}$ diameter and $>2.5 \mu \mathrm{m}$ deep air holes. The solid polymer infiltration result was directly inspected by cross-sectional scanning electron microscopy. It was observed that the holes are fully filled to the bottom. The photonic crystals were optically characterized by transmission measurements around the $1.5 \mu \mathrm{m}$ wavelength band both before and after infiltration. The observed high-frequency band edge shifts are consistent with close to $100 \%$ filling, for both the polymer and the liquid crystal. No differences were observed for filling under vacuum or ambient, indicating that the air diffuses efficiently through the liquid infiltrates, in agreement with estimates based on the capillary pressure rise.
\end{abstract}

Keywords: Photonic Crystal, infiltration, tuning, polymer, Liquid Crystal

\section{INTRODUCTION}

Photonic crystals ${ }^{1}$ are emerging as a new class of photonic devices, with great interest both for fundamental research on photon-matter interactions as for applications in photonic integrated circuits. Two-dimensional (2D) photonic crystals $(\mathrm{PhC})$ etched in planar waveguide structures offer currently the best manufacturing control, since they employ the sophisticated technology of state-of-the-art semiconductor lithography and processing. With proper designs, extremely good photon confinement can be realized even with such two-dimensional systems, as is demonstrated by the very high quality factor cavities already realized ${ }^{2}$. From their manufacturing, the $2 \mathrm{D} \mathrm{PhC} \mathrm{consist} \mathrm{naturally} \mathrm{of} \mathrm{a} \mathrm{dielectric,} \mathrm{usually} \mathrm{a}$ semiconductor, as the high-index material and air (or vacuum) as the low-index material. Since the refractive index of the semiconductor is typically rather large $(>3)$, it is possible to replace the air in the holes by another low-index functional material, thereby enhancing the possibilities of the $\mathrm{PhC}$, notably tuning through the low-index dielectric.

Several groups have already pursued the tuning of $2 \mathrm{D} \mathrm{PhC}$ by infiltrating the holes with a Liquid Crystal ${ }^{3-9}$, following the original suggestion by Busch and John ${ }^{10}$. While the tuning capabilities of Liquid Crystals are not likely to be surpassed by any other dielectric, their relatively slow response, anisotropy, or liquid state could make them unsuitable

${ }^{*}$ r.v.d.heijden@tue.nl

Tuning the Optic Response of Photonic Bandgap Structures III, edited by Paul V. Braun, Sharon M. Weiss, Proc. of SPIE Vol. 6322, 632205, (2006) · 0277-786X/06/\$15 · doi: 10.1117/12.679358 
for some applications. Moreover, even for purely passive applications, it is of interest to employ a third material as an infill to enhance the design parameters of device components ${ }^{11}$ or to decrease out of plane losses ${ }^{12}$. Therefore a need exists to search for other materials that can be easily infiltrated in the air holes. A smaller tunability range should be compensated by exploiting a nonlinearity-enhancing property of the photonic crystal like a cavity or a band edge. In this paper we report the filling of the holes with a Liquid Crystal as well as with a solid polymer achieved by infiltration of a liquid monomer and subsequent thermal polymerization. The solid state of the polymer allows for a detailed inspection of the filling process by cross-sectional Scanning Electron Microscopy (SEM) of cleaved samples.

\section{OPTICAL CHARACTERIZATION OF INFILTRATED SAMPLES}

Since the intended application domain of the present work is in the integrated circuits operating near the $1.5 \mu \mathrm{m}$ telecom wavelength, the material system is the InP-InGaAsP-InP planar waveguide, consisting of a $500 \mathrm{~nm}$ upper InP cladding and a $500 \mathrm{~nm} \mathrm{InGaAsP}$ core, grown by Metal-Organic Chemical Vapour deposition (MOCVD) lattice matched on an $\mathrm{InP}$ buffer. The low vertical index contrast between the core and the cladding $(\sim 3.4 \mathrm{vs} \sim 3.2)$ imposes a high aspect ratio etch of $>2.5 \mu \mathrm{m}$ deep, $\sim 200 \mathrm{~nm}$ diameter holes on a $\sim 400 \mathrm{~nm}$ pitch in a hexagonal pattern. Photonic crystal patterns were etched using a $\mathrm{Cl}_{2}$-based Inductively Coupled Plasma process, with addition of a small amount of $\mathrm{O}_{2}$ for sidewall passivation ${ }^{13}$.

The liquid monomer trimethylolpropane triacrylate (TMPTA, Aldrich), mixed with a thermal initiator (0.5\% AIBN, Azoisobutyronitrile, Fluka), was chosen as infiltrant ${ }^{14}$ for its ease of polymerization under thermal or UV treatment. Since the semiconductor is not transparent to UV-radiation, the thermal process was selected ${ }^{14}$. In a separate experiment, employing a thin layer of the polymer, its refractive index was determined from ellipsometry to be $n_{\text {inf }}=$ 1.465 at $1.5 \mu \mathrm{m}$ wavelength. Since wetting of the infiltrant with the semiconductor surface is crucial for infiltration, the wetting was first investigated from contact angle measurements. A rinse with dilute HF followed by one with propanol2 gave contact angles below $\sim 3^{0}$ and resulted in successful infiltration.

The photonic stopbands for TE-polarization were calculated in both $\mathrm{PhC}$ symmetry directions $\Gamma \mathrm{K}$ and $\Gamma \mathrm{M}$, using a commercial software package ${ }^{15}$ that employs a $2 \mathrm{D}$ plane wave expansion method ${ }^{16}$. The effective index of 3.25 of the planar waveguide was used in the 2D calculation. The results are shown in Fig. 1 for the design ratio of hole radius $r$ to

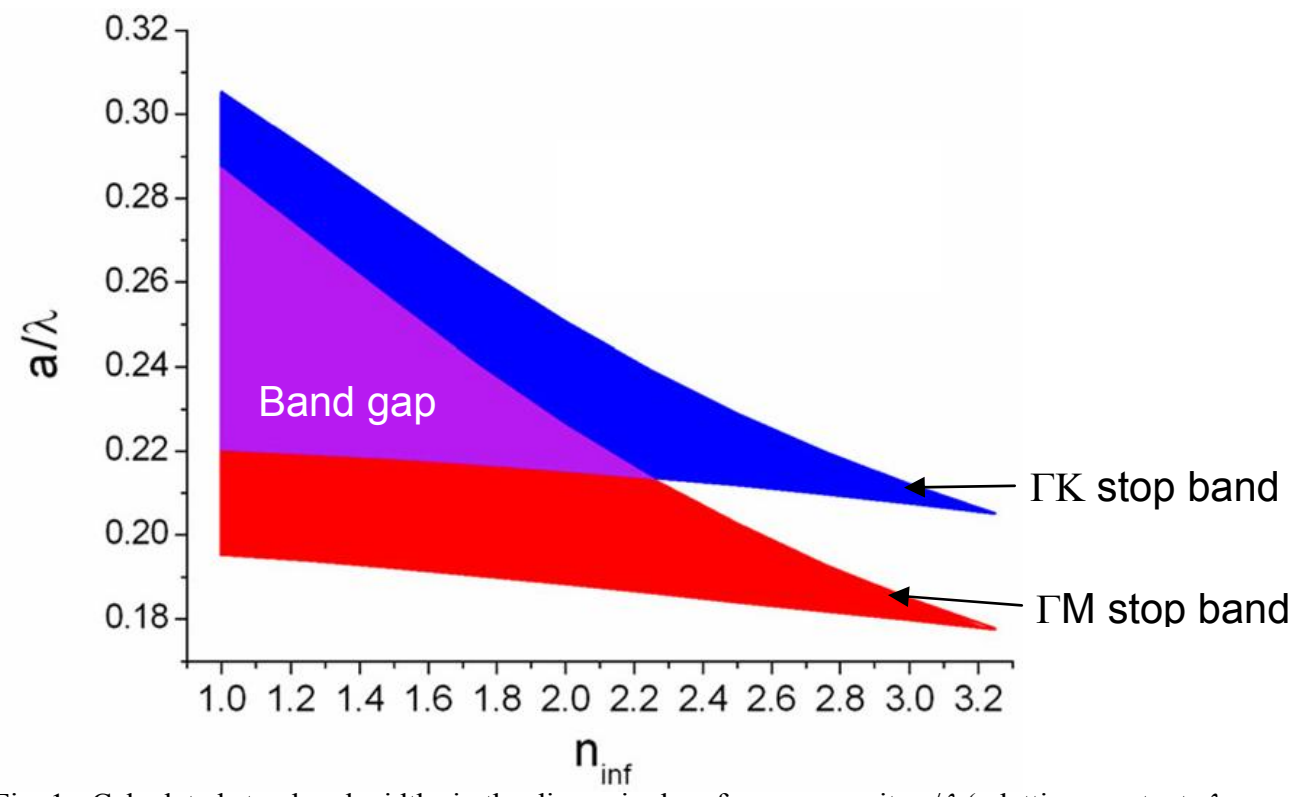

Fig. 1 Calculated stop band widths in the dimensionless frequency units $a / \lambda$ ( $a$ lattice constant, $\lambda$ wavelength) in the two crystallographic directions $\Gamma \mathrm{K}$ and $\Gamma \mathrm{M}$ as a function of the refractive index $n_{\text {inf }}$ of the infill material. Two-dimensional calculation, assuming host lattice effective refractive index of 3.25 . 


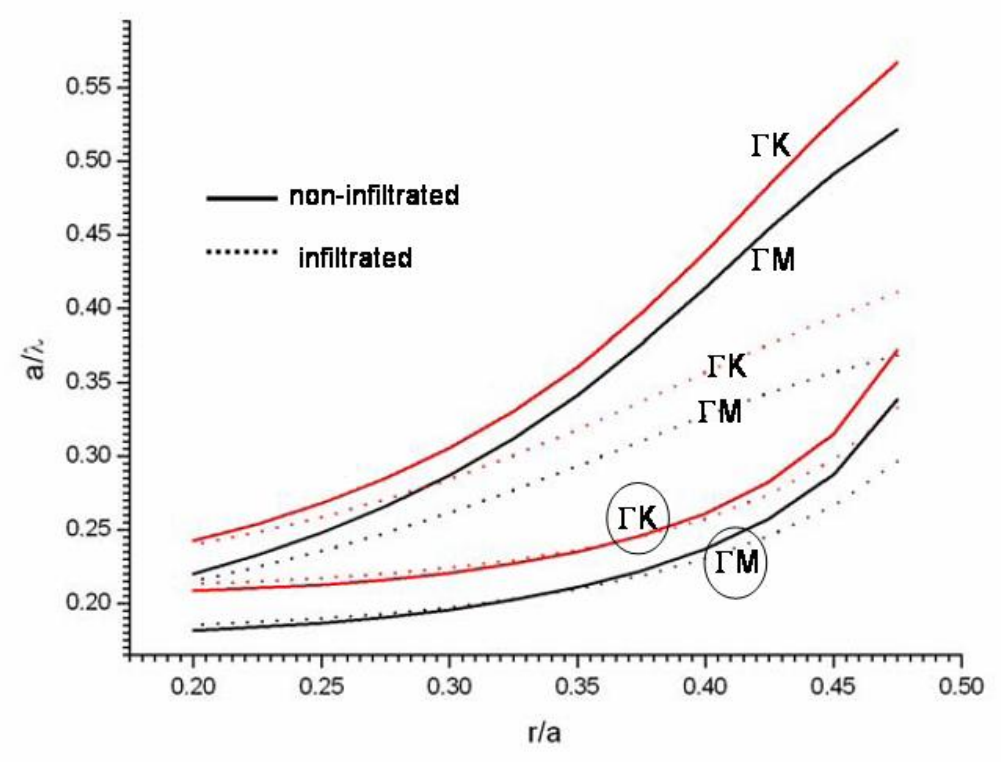

Fig. 2. Calculated stop band widths in the dimensionless frequency units $a / \lambda$ ( $a$ lattice constant, $\lambda$ wavelength) in the two crystallographic directions $\Gamma \mathrm{K}$ and $\Gamma \mathrm{M}$ as a function of the ratio $r / a$ with index $n_{\text {inf }}=1.456$. Two-dimensional calculation, assuming host lattice effective refractive index of 3.25 .

hexagonal lattice constant $a$ of 0.3. It follows that for the polymer index (1.465) the bandgap is reduced from the air value $\Delta(a / \lambda) /(a / \lambda) \sim 25 \%$ to $\sim 15 \%$ after filling, which is still conveniently large. Up to infiltrant indices of $\sim 2.3$ the gap remains in both directions.

Fig. 2 shows the upper and lower stopband edges (lowest bandgaps) in dimensionless units as a function of the hole radius to lattice constant ratio $r / a$. From this it should be noted that not only the bandwidths decrease when $r / a$ decreases, but also the relative effect of infiltration decreases with smaller hole radii.

The full calculated bandstructures for infiltrated $\left(n_{\text {inf }}=1.465\right)$ and empty photonic crystals are displayed in Fig. $3(a)$ and (b) using the 2D plane wave expansion method and effective host refractive index of 3.25 with design $r / a=0.3$. The calculations are shown for both polarizations. The effect of infiltration on the bandstructure is much stronger for the TE than for the TM polarization. This has the same origin as the absence of a bandgap for the TM-polarization at $r / a=0.3$. Figures $3(c)$ and $(d)$ show the measured transmission data in the $\Gamma$ K-direction. The data were obtained using a tunable laser (1470-1570 nm) and by combining data from crystals with varying $a$ but fixed $r / a$ etched on the same chip. Light was coupled in and out of simultaneously etched ridge waveguides using microscope objectives. For the TEpolarization, a substantial redshift of the high-frequency band edge was observed, with a magnitude in fair agreement with the calculated shift assuming the holes are completely filled with the $n=1.456$ dielectric. It is important to observe that the transmission in the high-frequency band $(a / \lambda>0.27)$ has not degraded after filling, and actually is improved. The reduction of the in-plane index contrast, while reducing the bandgap, is expected to have a beneficial effect on the out-of-plane losses. Fig. 3(d) shows transmission data for the TM polarization after infiltration, along with a calculated transmission. Data before infiltration are not available for this polarization. The calculation is a 3D FDTD using commercial software ${ }^{15}$ assuming straight cylindrical holes $3 \mu \mathrm{m}$ deep. Also in this polarization, the transmission is in good agreement with the calculations; in fact the measured suppression in the frequency range $a / \lambda \sim 0.21-0.28$ is larger than in the simulations. Although no real gap exists in the $\Gamma \mathrm{K}$ direction for TM-polarization, the symmetry of the mode of the second lowest band is such that it is not excited experimentally and also not using the mode exciter used for the 

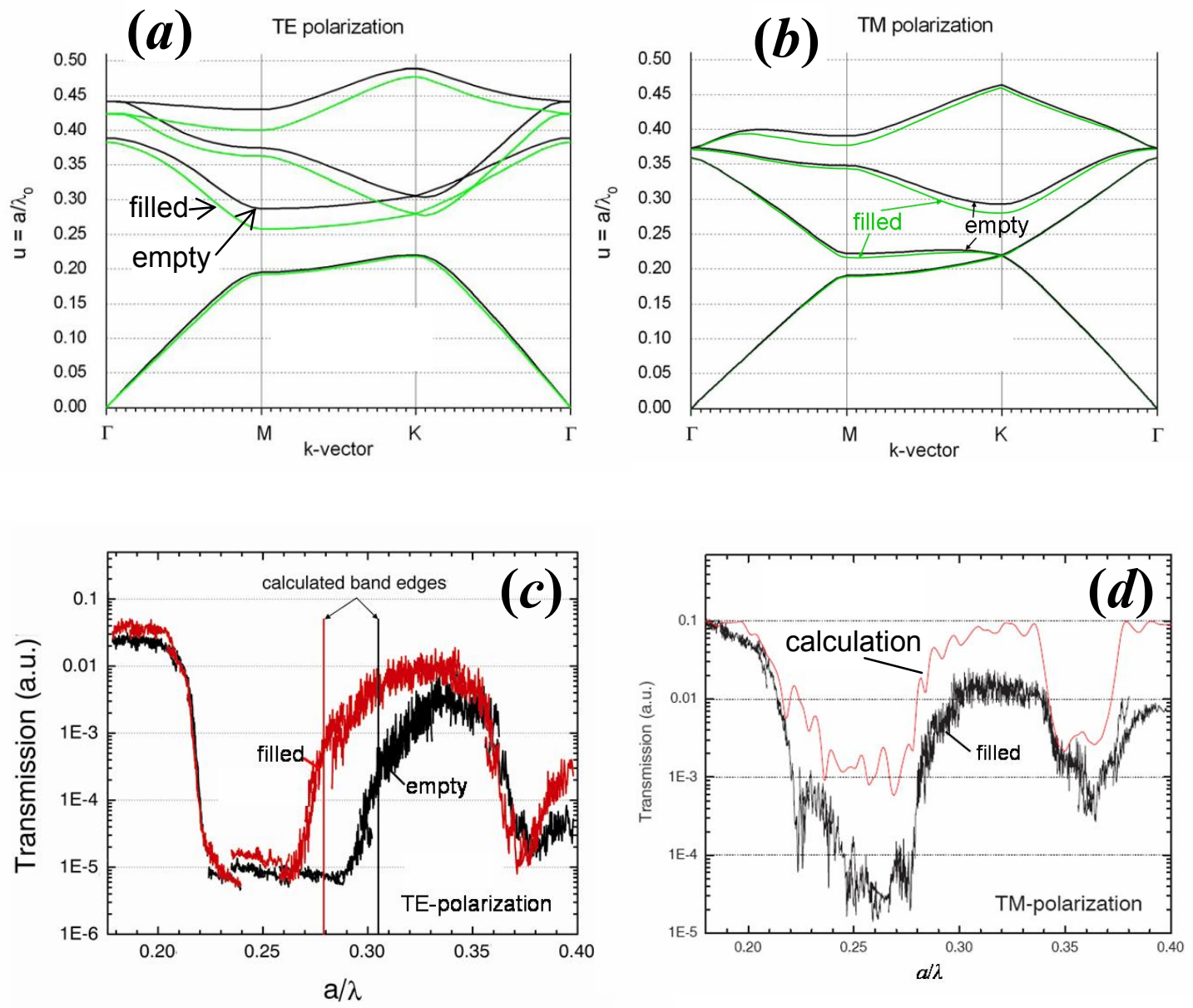

Fig. 3 (a) Two-dimensional band diagram calculation using a plane wave expansion method for an empty and filled $\left(n_{\text {inf }}=1.465\right)$ photonic crystal; hole radius to lattice constant ratio $r / a=0.3$; TE-polarization; host lattice effective refractive index of 3.25. (b) Same as (a), but for TM-polarization.

(c) Measured transmission along $\Gamma \mathrm{K}$-direction before ("empty") and after ("filled") filling with the polymer for TEpolarization. (d) Measured and calculated (3D FDTD) transmission along $\Gamma \mathrm{K}$-direction for TM-polarization. (e) Cross-sectional SEM-image of typical photonic crystal hole

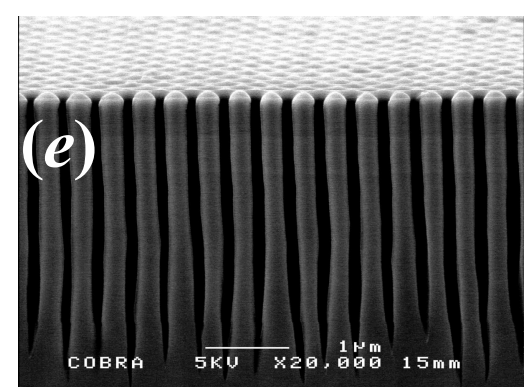
pattern (though not the same as used for $(\boldsymbol{c})$ and $(\boldsymbol{d})$

simulations ${ }^{17}$. A SEM cross-section of a typical etched hole pattern is shown in Fig. 3(e), albeit not for the same crystal from which the transmission data were taken.

\section{CROSS-SECTIONAL SEM CHARACTERIZATION}

Since the polymer is a solid, it is possible to inspect directly the filling. For this purpose the filled crystals were cleaved and the cross-sections examined with a SEM. Typical results for different feature sizes are displayed in Fig. 4, obtained from samples from etch-test runs and so of inferior shape. Even for the smallest sizes $(<200 \mathrm{~nm}, 4(c))$, the holes are entirely filled down to the bottom. The infill accurately follows shape irregularities (e.g. 4(a)) or bottom roughness 

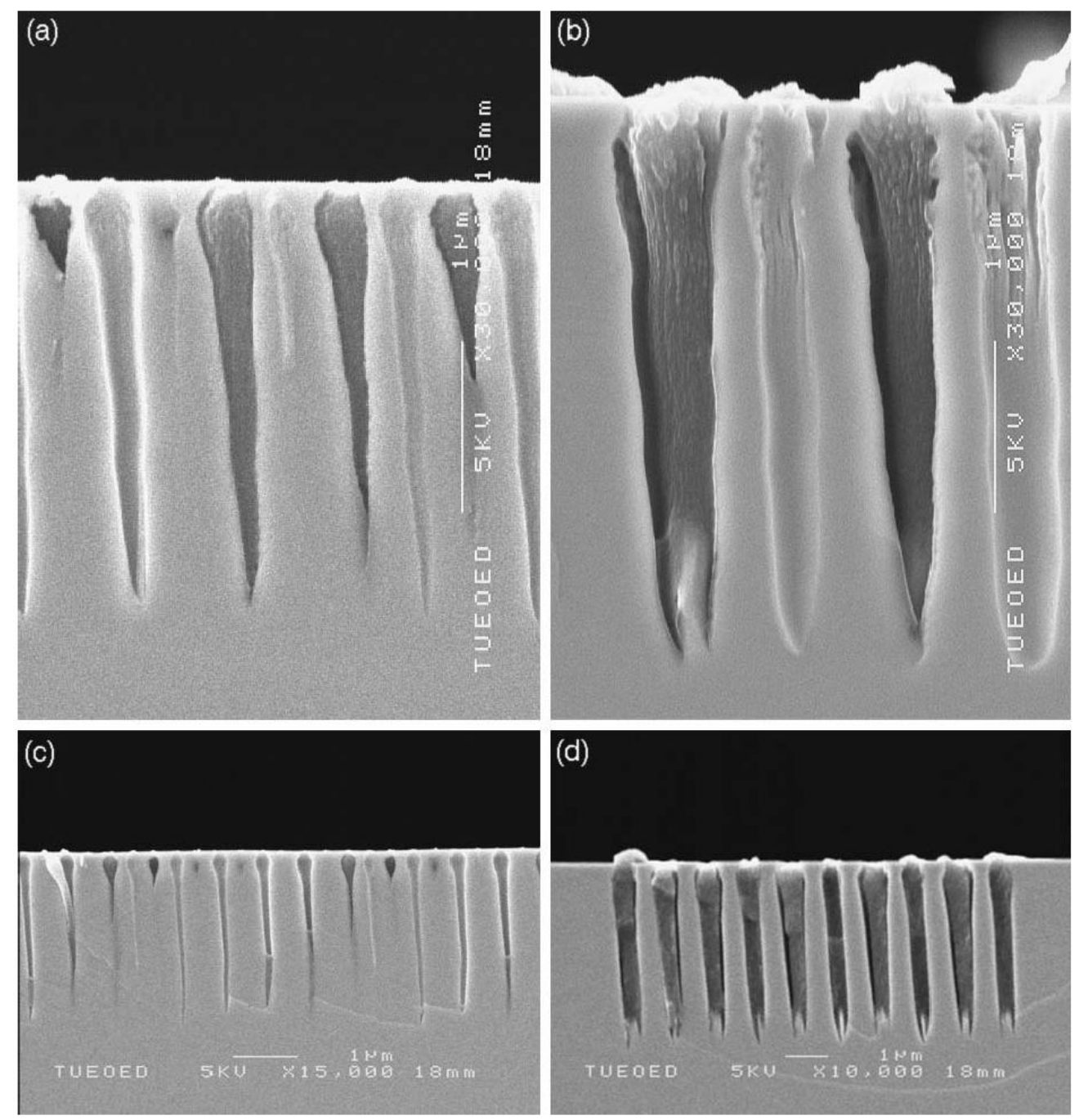

Fig. 4 Cross-sectional SEM-images of photonic crystals with polymer-filled holes. The hole patterns were taken from etch-test runs having poor hole shapes, not representative for the crystals used for optical characterization. The liquid monomer was infiltrated under vacuum ( 20 mbar). (a) Intentional hole diameter $240 \mathrm{~nm}$ (b) Intentional hole diameter $480 \mathrm{~nm}(\boldsymbol{c})$ Intentional hole diameter $180 \mathrm{~nm}(\boldsymbol{d})$ Trenches with intended width $480 \mathrm{~nm}$.

$(4(d))$. It is also seen (particularly well from $4(b))$ ), that a partial detachment from the wall may occur and consequent incomplete filling. This is not attributed to incomplete liquid infiltration, but to volume shrinkage upon polymerization, which is known to be of the order of $10 \%$ for these type of polymerizations.

Some of the holes in Fig. 4 appear empty, which could suggest that only a fraction of the holes are filled. To investigate this in detail, for one sample both sides of the cleaved piece were examined under the SEM, thereby carefully identifying the corresponding holes. It is not expected that the tiny polymer plugs in the holes would be cleaved as well, so they should remain at either side. Since the crystal is cleaved under a small $\left(\sim 3^{0}\right)$ angle with respect to the $\Gamma \mathrm{K}$ photonic crystallographic axes, they should periodically reside in the one or in the other half, as is suggested by the inset 


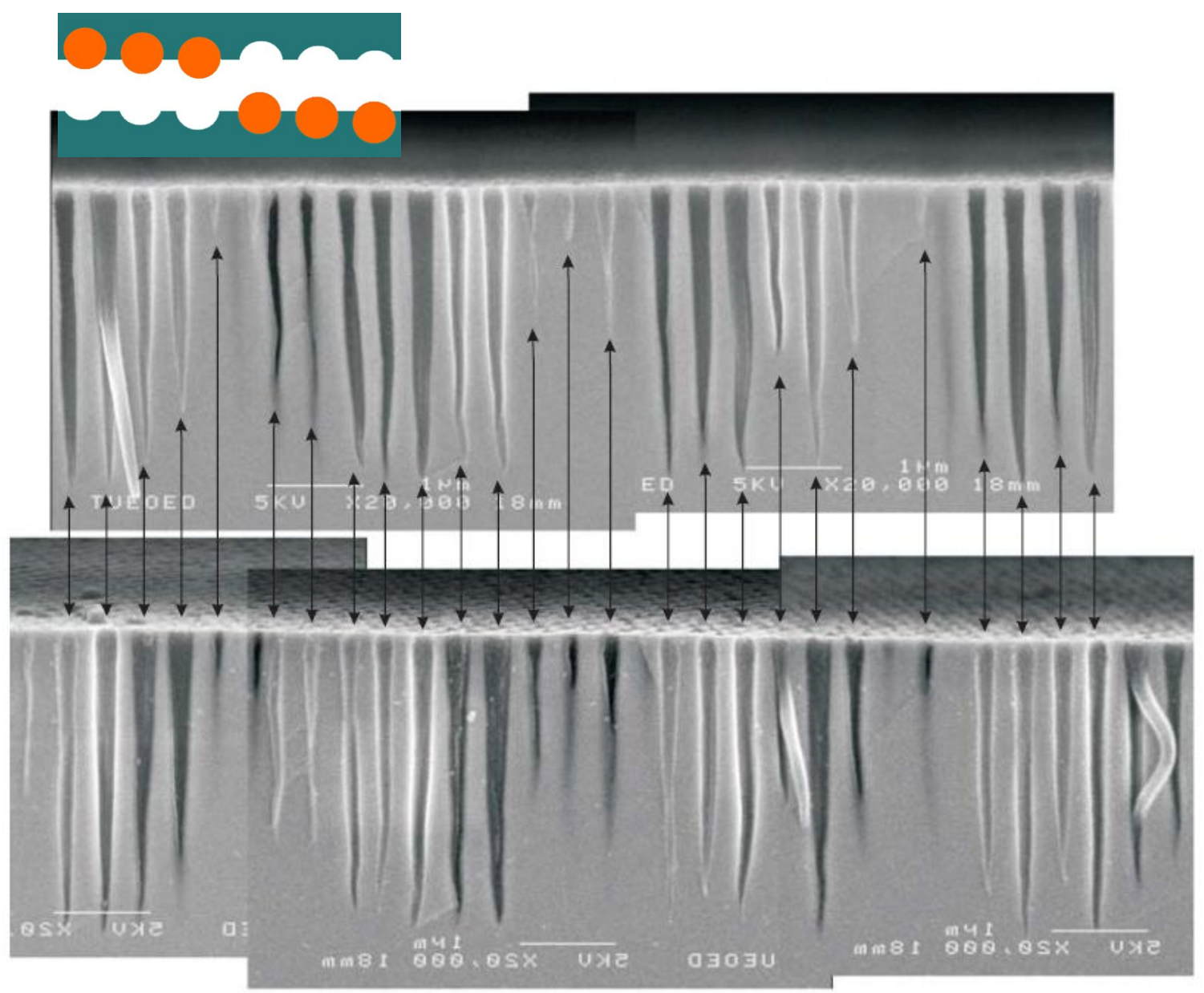

Fig. 5 Comparison of the two opposite pieces of a cleaved InP sample with an etched hole pattern. The arrows identify the same holes in the two pieces. The cleave is made under a small angle with respect to the $Г \mathrm{~K}$ photonic crystallographic direction. The polymer plug is present in either of the two sides. The inset on top schematically illustrates the situation.

in Fig. 5. The experimental observations from both sides confirm this expectation as is evidenced by Fig. 5. From the data in Figs. 4 and 5 it can be concluded that SEM-inspection proofs the complete infiltration of the holes.

\section{LIQUID CRYSTAL INFILTRATION}

The infiltrations with the liquid monomer were performed both under modest evacuation (residual pressure $\sim 20$ mbar) as well as under ambient atmosphere. No systematic changes were observed dependent on the conditions, neither from the optical data, nor from the cross-sectional SEM images. Apparently, degassing of the holes is very efficient since no effects from trapped air are found. A possible route is the diffusion of the air through the liquid plug during the infiltration, driven by the quite high capillary pressure (up to $\sim 5$ bar if there would be no degassing). Although the gas permeability of the used liquid was not known, using typical data for polymers suggested that this route is indeed plausible, leading to degassing times $(<1 \mathrm{~s})$, short compared to the time scale of the infiltration and subsequent polymerization process $\left(>10^{3} \mathrm{~s}\right)^{18}$. To show whether infiltration under ambient works also for the Liquid Crystals (LC) that are usually infiltrated under vacuum, a sample was infiltrated with the common nematic Liquid Crystal known as 5 $\mathrm{CB}$ or K-15 (4-n-pentyl-4-cyanobiphenyl). The clearing point of this $\mathrm{LC}$ is $\sim 35{ }^{\circ} \mathrm{C}$, and it was infiltrated at room 

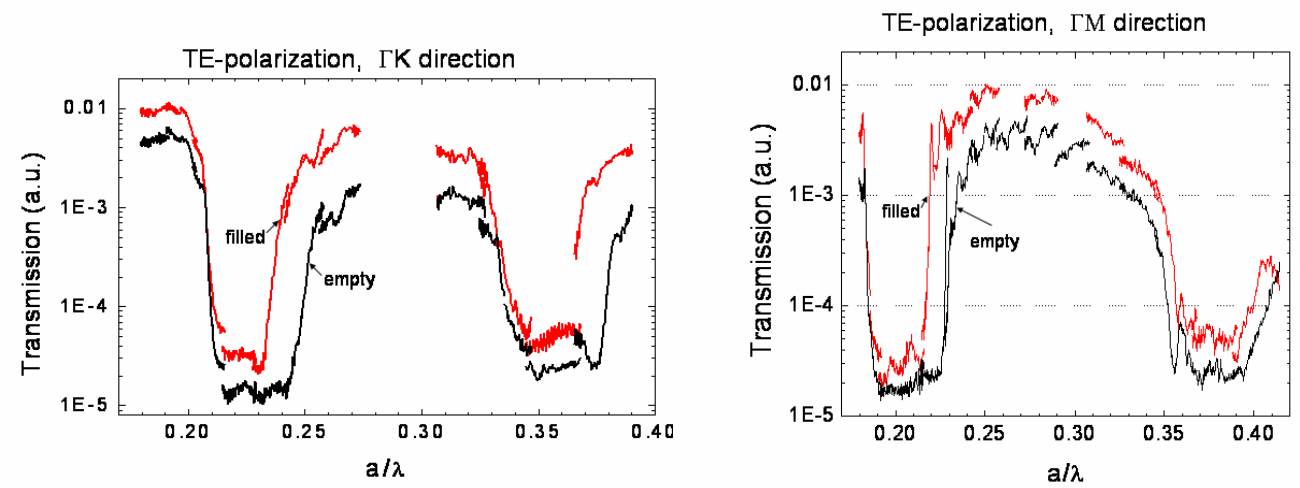

Fig. 6 Measured transmission of a photonic crystal before ("empty") and after ("filled") infiltration with Liquid Crystal 5CB in the ГK-direction (left) and ГМdirection (right); TE-polarization in both cases.

temperature $\left(\sim 25^{\circ} \mathrm{C}\right)$ and ambient atmosphere. Some preliminary transmission results are shown in Fig. 6 . To obtain an accurate number for the filling fraction is not straightforward, in view of the uncertainty of the refractive index ${ }^{5,19}$. The ordinary and extraordinary refractive index are 1.516 and 1.682 respectively ${ }^{19}$, but the orientation distribution of the LCdirector is not known. The photonic crystal in Fig. 6 accidentally had a smaller $r / a$ ratio than intended, which makes it less sensitive to infiltration (see Fig. 2). Nevertheless, the substantial shifts in Fig. 6 indicate that the filling fraction also must be close to complete.

\section{CONCLUSION}

It has been shown from optical data and direct cross-sectional SEM-investigation that the airholes in a deeply etched two-dimensional photonic crystal can be easily filled with a solid polymer. The filling was based on liquid infiltration with a monomer and subsequent thermal polymerization. Filling can be accomplished under ambient conditions which appears to work also for infiltration with liquid crystal.

\section{ACKNOWLEDGEMENTS}

The authors would like to thank P.Nouwens, R. van Veldhoven, E.J. Geluk, T. de Vries, M. Sander, and E. Smalbrugge for their contributions to the experimental work and J. van der Tol, A. Kok, S. Oei, J. Haverkort, and A. Silov for helpful discussions. Part of this research is supported by NanoNed, a technology programme of the Dutch ministry of Economic Affairs.

\section{REFERENCES}

${ }^{1}$ J.D. Joannopoulos, R.D. Meade, and J.N. Winn, Photonic crystals, molding the flow of light (Princeton University Press, 1995)

${ }^{2}$ T. Asano, B-S. Song, and S. Noda, Opt. Express 14, 1996 (2006)

${ }^{3}$ S.W. Leonard, J.P. Mondia, H.M. van Driel, O. Toader, S. John, K. Busch, A. Birner, U. Gösele, and V. Lehman, Phys. Rev. B 61, R2389 (2000)

${ }^{4}$ Ch. Schuller, F. Klopf, J.P. Reithmaier, M. Kamp, and A. Forchel, Appl. Phys. Lett. 82, 2767 (2003)

${ }^{5}$ J. Martz, B. Wild, R. Ferrini, L.A. Dunbar, M. Mulot, S. Anand, R. Houdré, and L. Zuppiroli, Proc. of SPIE 5926, 592601, (2005)

${ }^{6}$ B. Maune, M. Lončar, J. Witzens, M. Hochberg, T. Baehr-Jones, D. Psaltis, A. Scherer, and Y. Qiu, Appl. Phys. Lett. 85, 360 (2004) 
${ }^{7}$ B. Maune, J. Witzens, T. Baehr-Jones, M. Kolodrubetz, H. Atwater, A. Scherer, R. Hagen, and Y. Qiu, Optics Express 13, $4699(2005)$

${ }^{8}$ G. Mertens, T. Röder, H. Matthias, H. Marsmann, H.S.R. Kitzerow, S. Schweizer, C. Jamois, R.B. Wehrspohn, and M. Neubert, Appl. Phys. Lett. 83, 3036 (2003)

${ }^{9}$ Ch. Schuller, J.P. Reithmaier, J. Zimmermann, M. Kamp, A. Forchel, and S. Anand, Appl. Phys. Lett. 87, 121105 (2005)

${ }^{10}$ K. Busch, and S. John, Phys. Rev. Lett. 83, 967 (1999)

${ }^{11}$ S. Mingaleev, M. Schillinger, D. Hermann, and K. Busch, Opt. Lett. 29, 2858 (2004)

${ }^{12}$ M.M. Raj, S. Toyoshima, and S. Arai, Jpn. J. Appl. Phys. 38, L811 (1999)

${ }^{13}$ C.F. Carlström, R. van der Heijden, F. Karouta, E. van der Drift, R.W. van der Heijden, H.W.M. Salemink, J. Vac. Sci. Technol. B 24, L6 (2006)

${ }^{14}$ R. van der Heijden, C.F. Carlström, J.A.P. Snijders, R.W. van der Heijden, F. Karouta, R. Nötzel, H.W.M. Salemink, B.K.C. Kjellander, C.W.M. Bastiaansen, D.J. Broer, and E. van der Drift, Appl. Phys. Lett. 88, 161112 (2006)

${ }^{15}$ CrystalWave from Photon Design, UK

${ }^{16}$ M. Plihal and A.A. Maradudin, Phys. Rev. B 44, 8565 (1991)

${ }^{17}$ T.F. Krauss, R.M. De La Rue, and S. Brand, Nature 383, 699 (1996)

${ }^{18}$ R. van der Heijden, B.K.C. Kjellander, C.F. Carlström, J.A.P. Snijders, R.W. van der Heijden, C.W.M. Bastiaansen, D.J. Broer, F. Karouta, R. Nötzel, E. van der Drift, and H.W.M. Salemink, Proc. SPIE-Int. Soc. Opt. Eng. 6182, 213 (2006)

${ }^{19}$ R. Ferrini, J. Martz, L. Zuppiroli, B. Wild, V. Zabelin, L.A. Dunbar, R. Houdre, M. Mulot, and S. Anand, Opt. Lett. 31, 1238 (2006) 